\title{
Statistical Models and the Theory of Hypothesis Testing in Medicine
}

\author{
Submitted 20/08/19, 1st revision 18/09/19, 2nd revision 14/10/19, accepted 29/11/19
}

\author{
Gazaryan V.A., ${ }^{1}$ Guryanova I.E., ${ }^{2}$ Melekhina T.L. ${ }^{3}$ \\ Abstract:
}

Purpose: The purpose of this work is to develop statistical approaches for treatment efficiency analysis on a medical case. These methods include the effect evaluation method performed with various preparations in regards to several factors such as the patient's blood state and his/her biochemical values.

Design/Methodology/Approach: The results of this survey were divided into three groups: the first included the blood biochemistry values before the test, the second - an hour after the test, the the third - two hours after the test. When visually comparing the results of the analyses of the three groups, it was possible to assume that the load of FLC did not significantly affect the biochemistry of the patients' blood. To test this medical assumption, various statistical criteria of the theory of testing statistical hypotheses have been applied.

Findings: Statistical analysis of changes in the level of lipase and triglycerides in the biochemical analysis of the blood of patients with chronic pancreatitis after ingestion of food containing medium-chain fatty acids showed that there is no overall disruption in the functioning of the pancreas.

Practical implications: Clinical practice has shown that more than $85 \%$ of patients tolerated testing with medium chain fatty acids, not experiencing a painful abdominal symptom and other negative consequences of a violation of external secretion of the pancreas.

Originality/Value: In clinical practice, the Russian Academy of Medical Sciences for the first time took into account the results of a statistical analysis.

Keywords: Mathematical statistics, theory of statistical hypothesis testing, statistical criterion.

JEL Code: C12, C15, I10, I12.

Paper type: Research article: Mathematical Statistics.

\section{Acknowledgement:}

The work is executed at partial financial support of RFBR (grants 17-07-00832, 18-07-00424).

\footnotetext{
${ }^{1}$ Associate Professor of the Department of Data Analysis, Decision-Making and Financial Technology of Financial University under the Government of the Russian Federation, Moscow and Researcher of the Department of Mathematical Modeling and Computer Science of M.V. Lomonosov Moscow State University VAGazaryan@fa.ru ${ }^{2}$ Associate Professor of the Department of Data Analysis, Decision-Making and Financial Technology of Financial University under the Government of the Russian Federation, Moscow, IEGuryanova@fa.ru

${ }^{3}$ Associate Professor of the Department of Data Analysis, Decision-Making and Financial Technology of Financial University under the Government of the Russian Federation, Moscow, TMelehina@fa.ru
} 


\section{Introduction}

For medical practices it is important to understand the possibility of energy nutrition of patients with chronic pancreatitis experiencing protein-energy malnutrition. In gastroenterology for this purpose it is considered to prefer products, which contain medium-chain fatty acids to foods with vegetable or animal fats which are longchained and negatively affect the pancreas of patients with chronic pancreatitis.

Gastroenterologists Clinic Institute of nutrition Russian Academy of medical Sciences suggested checking the tolerability of the patients with chronic pancreatitis and fat load with medium chain fatty acids. The conducted test also allows to determine the presence of exocrine insufficiency in the pancreas when administered in the diet products with medium chain fatty acids based on criteria such as increasing the number of triglycerides and the level of lipase in the blood of patients in relation to the original condition.

After the necessary biochemical tests, the results of the survey were divided into three groups: first were the values of blood chemistry before the test, second one hour after the test, and the third - two hours after the test. By visual comparison of the results of the analyses of the three groups it can be assumed that the load chain fatty acids did not significantly affect the blood chemistry of patients. With what degree of reliability can we speak about the load test acceptable tolerability among the patients with chronic pancreatitis? How significant is this result? What is the best way to quantify the differences between the biochemical value levels? On the one hand, these differences may be of regular character, but visually indistinguishable, on the other hand, they may be random. To determine if there is any effect of lipid load in the current statistical ensemble we will apply mathematical statistical models and the theory of statistical hypothesis testing.

\section{The Statistical Method}

Let $(\Omega, P(\Omega), \operatorname{Pr}(\cdot, \theta)), \theta \in \Theta$ - to be a family of probability spaces of stochastic experiment and due to the observation of $\omega \in \Omega$ the conclusion about the parameter value $\theta \in \Theta$ is to be drawn (Pyt'ev, 2018). Suppose, for example, we need to test the hypothesis that $\theta=\theta_{0}$ with the alternative that $\theta=\theta_{1} \neq \theta_{0}$. Let's denote $\Phi\left(\theta_{0}, \theta_{1}, p r\right) \in \Omega$ as the region of acceptance of hypotheses where the criterion meets the most powerful level of trust $p r$ : if $\omega \in \Phi\left(\theta_{0}, \theta_{1}, p r\right)$ then the hypotheses is to be accepted, if $\quad \omega \notin \Phi\left(\theta_{0}, \theta_{1}, p r\right)$ it is to be rejected. The probability to accept the hypothesis when it is actually true is $\operatorname{Pr}\left(\omega \in \Phi\left(\theta_{0}, \theta_{1}, p r\right), \theta_{0}\right)=p r \in[0,1]$.

The region $\Omega \backslash \Phi\left(\theta_{0}, \theta_{1}, p r\right)$ is called critical and the probability of mistakenly rejecting the idea is $\operatorname{Pr}\left(\omega \notin \Phi\left(\theta_{0}, \theta_{1}, p r\right), \theta_{0}\right)=1-p r=\alpha$ is called the level of criterion; the probability of rejecting the hypothesis when alternative is true is called the power of the criterion. The regions $\Phi\left(\theta_{0}, \theta_{1}, p r\right)$ corresponding to the most powerful criterion level $p r$ corresponds to the largest probability of rejecting the 
hypothesis when alternative is true, i.e. the smallest probability of an erroneous acceptance of the hypothesis in comparison with other areas $\left(\widetilde{\Phi}\left(\theta_{0}, \theta_{1}, p r\right)\right.$ of the same level $\operatorname{pr}: \operatorname{Pr}\left(\omega \in \Phi\left(\theta_{0}, \theta_{1}, p r\right), \theta_{1}\right) \leq \operatorname{Pr}\left(\omega \in \widetilde{\Phi}\left(\theta_{0}, \theta_{1}, p r\right), \theta_{1}\right)$.

The region $\Phi\left(\theta_{0}, \theta_{1}, p r\right)$ has also the property of indisplacement: the probability to accept the true hypothesis is not less than the probability to accept it mistakenly: $\operatorname{Pr}\left(\omega \in \Phi\left(\theta_{0}, \theta_{1}, p r\right), \theta_{1}\right) \leq p r \in[0,1]$.

For the family of pairs a hypothesis-alternative $\left(\theta_{0}, \theta_{1}\right) \in Z$ where $Z \subset \Theta \times \Theta$ is a symmetric anti-reflective ratio which specifies the one-to-one mapping $\Theta \rightarrow \Theta$ for each $p r \in[0,1]$ в $\Omega \times \Theta$ in (Gazaryan, 2006) is the defined discriminant variety

$$
D_{p r}=\left\{\left(\omega, \theta_{0}\right) \in \Omega \times \Theta, \omega \in \Phi\left(\theta_{0}, \theta_{1}, p r\right),\left(\theta_{0}, \theta_{1}\right) \in Z\right\}=\left\{\left(\omega, \theta_{0}\right) \in \Omega \times \Theta,\right.
$$
$\left.\omega \in \Phi\left(\theta_{0}, p r\right)\right\}$,

where

$\left.\Phi\left(\theta_{0}, p r\right) \equiv \Phi\left(\theta_{0}, \theta_{1}, p r\right)\right|_{\left(\theta_{0}, \theta_{1}\right) \in Z}, \theta_{0} \in \Theta$.

For each $\theta \in \Theta$ it is defined the discriminant variety of the level of trust $r \in[0,1]$ $\Phi^{-1}(\omega, p r)=\left\{\theta_{0} \in \Theta,\left(\omega, \theta_{0}\right) \in D_{p r}\right\}$. So for every $\left(\theta_{0}, \theta_{1}\right) \in Z, p r \in[0,1], \theta \in \Theta$ $\operatorname{Pr}\left(\omega \in \Phi\left(\theta_{0}, \theta_{1}, p r\right), \theta\right) \equiv \operatorname{Pr}\left(\omega \in \Phi\left(\theta_{0}, p r\right), \theta\right)=\operatorname{Pr}\left(\theta_{0} \in \Phi^{-1}(\omega, p r), \theta\right)$ and $\operatorname{Pr}\left(\theta_{0} \in \Phi^{-1}(\omega, p r), \theta_{0}\right)=\operatorname{pr}, \theta_{0} \in \Theta$.

The random variety $\Phi^{-1}(\omega, p r)$ covers the true value of parameter of the distribution $\theta_{0} \in \Theta$ with the probability $p r \in[0,1]$. The variety $\Phi^{-1}(\omega, p r)$ is different from other evaluating varieties $\widetilde{\Phi}^{-1}(\omega, p r)$ of level $p r$ in

$\operatorname{Pr}\left(\theta_{0} \in \Phi^{-1}(\omega, p r), \theta_{1}\right) \leq \operatorname{Pr}\left(\theta_{0} \in \widetilde{\Phi}^{-1}(\omega, p r), \theta_{1}\right),\left(\theta_{0}, \theta_{1}\right) \in Z$,

and the property of indisplacement

$\operatorname{Pr}\left(\theta_{0} \in \Phi^{-1}(\omega, p r), \theta_{1}\right) \leq p r$.

So $\Phi^{-1}(\omega, p r)$ is the variety of such $\theta_{0} \in \Theta,\left(\theta_{0}, \theta_{1}\right) \in Z$ in which the hypothesis $\theta=\theta_{0}$ is accepted if $\omega \in \Omega ; \Phi\left(\theta_{0}, \theta_{1}, p r\right)$ is observed in experiment as a variety of such results of $\omega \in \Omega$ in which the hypothesis $\theta=\theta_{0}$ is accepted.

Since $\Phi^{-1}(\omega, p r) \subset \Phi^{-1}\left(\omega, p r^{\prime}\right), \omega \in \Omega$, and if $p r<p r^{\prime}$ that the more minimum $p r \in[0,1]$, in which $\theta_{0} \in \Phi^{-1}(\omega, p r)$ the less trust to the inference that $\theta_{0} \in \Theta$ is the true value of the parameter of the distribution and the lower the possibility of equality $\theta=\theta_{0}$. From inequality (3) it follows that the probability of the event $\theta_{0} \in \Phi^{-1}(\omega, p r)$ in condition of true hypothesis is no less than the correct alternative, and from the inequality (2) it implies that the event $\theta_{0} \in \Phi^{-1}(\omega, p r)$ probability with the true 
alternative is not more than the probability of inclusion $\theta_{0}$ in any confidence variety $\widetilde{\Phi}^{-1}(\omega, p r)$ of the same level (Gazaryan 2005).

Let us put forward the main hypothesis $H_{0}$ that load of medium chain fatty acids did not significantly affect the biochemical blood test of patients that is the values of triglycerides and lipase have not changed in the average. As an alternative hypothesis $H_{1}$, let us consider the statement that such changes could take place in blood samples of the patients. From the point of view of the theory of statistical hypothesis testing we are talking about verifying the hypothesis of equality of mathematical expectations of the dependent populations, as the patients included in the sample (group) are the same, and their grouping characteristics are connected with their testing by mediumchain fatty acids. If the main hypothesis is confirmed, it can be concluded on a good assimilation of medium chain fatty acids by pancreas. For the correct solution of the tasks it is previously required to test the hypothesis about equality of variances of the studied aggregates.

Because the clinical tests were conducted on the sample of size 30, i.e., a sufficiently small sample, along with the Student's test, which is sufficiently stable in the deviations from the normal distribution, we used nonparametric criteria for testing hypotheses about the equality of the characteristics of statistical aggregate for testing hypotheses, and then a comparison of the results was made.

Let us consider the independent random variables $\omega_{1}, \omega_{2}, \ldots, \omega_{n}$, characterizing the values of the studied component $n$ in biochemical blood tests of patients before testing on medium-chain fatty acids, then denote the values of the same component after testing as $\chi_{1}, \chi_{2}, \ldots, \chi_{n}$. Assume initially that the samples of the aggregates $\omega_{1}, \omega_{2}, \ldots, \omega_{n}$ and $\chi_{1}, \chi_{2}, \ldots, \chi_{n}$ obey the normal distribution with equal dispersions. As it was mentioned before, in the analysis of the influence of medium-chain fatty acids on functioning of the pancreas the biochemistry blood tests of the same patients but taken at different times are examined, thus sampling $\omega_{1}, \omega_{2}, \ldots, \omega_{n}$ and $\chi_{1}, \chi_{2}, \ldots, \chi_{n}$ are not independent.

In order to apply Student's criterion, it is necessary to form a sample of independent random variables $z_{i}=\chi_{i}-\omega_{i}, i=1, \ldots, n$. Then $z_{i}$ characterizes the difference between the results of patient's " $i$ " biochemical blood components before and after testing, that is, the change of biochemical index related to the patient's initial individual characteristics is excluded. Present $z_{i}$ as $z_{i}=\theta+e_{i} i=1, \ldots, n$, where $e_{1}, \ldots, e_{n}$ independent random variables distributed according to the normal law with zero mathematical expectation and dispersion $\sigma^{2}, \theta$ - the constant component of the variable changes of biochemical components that reflect the impact of medium-chain fatty acids on functioning of the pancreas. In the beginning we will check the main hypothesis $H_{0}: \theta=\theta_{0}$ against the alternative hypothesis $H_{1}: \theta \neq \theta_{0}$. Since the results of testing the hypothesis about equality of dispersions show that the dispersions of the studied complexes $\omega_{1}, \omega_{2}, \ldots, \omega_{n}$ and $\chi_{1}, \chi_{2}, \ldots, \chi_{n}$ can be considered as equal at the 
level of importance 0,01 (Pyt'ev, 2014), but $D z_{i}=D\left(\theta+e_{i}\right)=\sigma^{2}$ is unknown let us use Student's criterion for solving the problem of testing the hypothesis $H_{0}: \theta=\theta_{0}$ against alternative hypothesis $H_{1}: \theta \neq \theta_{0}$ which allows to construct evenly the most powerful unbiased region $\Phi\left(\theta_{0}, p r\right)$ (1) of making the main hypothesis based on Nayman-Pirson's lemma (Gazaryan, 2014):

$\Phi\left(\theta_{0}, p r\right)=\left\{z_{1}, z_{2}, \ldots, z_{n},\left|\frac{\bar{z}-\theta_{0}}{s / \sqrt{n}}\right| \leq \tau_{n-1}(p r)\right\}$ where $\bar{z}=\frac{1}{n} \sum_{j=1}^{n} z_{i}$,

$s^{2}=\frac{1}{n-1} \sum_{j=1}^{n}\left(z_{j}-\bar{z}\right)^{2}$

is an unbiased rate of dispersion, and $p r=\int_{-\tau_{n-1}(p r)}^{\tau_{n-1}(p r)} \rho(t) d t, \rho(t)$ is the density of Student's distribution with $n-1$ degree of freedom. Then the minimum value of the probability of mistakenly rejecting the hypothesis when observing the sample $z_{1}, \ldots, z_{n}$ is (Gazaryan, 2015)

$p=1-\inf \left\{\operatorname{pr}|p r \in[0,1],| \frac{\bar{z}-\theta_{0}}{s / \sqrt{n}} \mid \leq \tau_{n-1}(p r)\right\}=2 \int_{-\infty}^{-\left|t_{\text {observed }}\right|} \rho(t) d t$

where $t_{\text {observed }}=\frac{\bar{z}-\theta_{0}}{s / \sqrt{n}}$.

\section{Software Implementation Results}

As it was mentioned earlier, the survey results of thirty patients were divided into three groups: the first included the results of blood biochemistry before the test, second one hour after the test, in the third - two hours after testing.

A computational experiment was conducted using programmatic means of system data analysis STATISTICA. At the beginning the main hypothesis $H_{\mathrm{T} 0}: \theta_{\mathrm{T}}=0$ was nominated, meaning that the number of triglycerides in the blood of patients has not changed on the average under the influence of medium-chain fatty acids. The results of applying the Student's test for samples of values of triglycerides in an hour and two hour after the test patients is shown in table 1 and table 2 , respectively.

Table 1. The results of applying the Student's test for samples of values of triglycerides in an hour after the test patients.

\begin{tabular}{|l|l|l|l|l|l|l|}
\hline \multirow{2}{*}{ Zero hypothesis } & \multicolumn{5}{|l|}{ T-test for Dependent Samples } \\
\cline { 2 - 7 } & Mean & Std. Dv. & $\mathrm{N}$ & $\mathrm{t}$ & $\mathrm{df}$ & $\mathrm{p}$ \\
\hline $\begin{array}{l}\text { triglycer. on an empty } \\
\text { stomach }\end{array}$ & 1,425217 & 0,793410 & & & & \\
\hline $\begin{array}{l}\text { triglycerides after an } \\
\text { hour }\end{array}$ & 1,390000 & 0,800477 & 23 & 1,018844 & 22 & 0,319351 \\
\hline
\end{tabular}


Table 2. The results of applying the Student's test for samples of values of triglycerides in an two hours after the test patients.

\begin{tabular}{|l|l|l|l|l|l|l|}
\hline Zero hypothesis & \multicolumn{6}{|l|}{ T-test for Dependent Samples } \\
\cline { 2 - 8 } & Mean & Std. Dv. & $\mathrm{N}$ & $\mathrm{t}$ & $\mathrm{df}$ & $\mathrm{p}$ \\
\hline $\begin{array}{l}\text { triglycer. on an } \\
\text { empty stomach }\end{array}$ & 1,425217 & 0,793410 & & & & \\
\hline $\begin{array}{l}\text { triglycerides after } \\
\text { two hours }\end{array}$ & 1,527826 & 0,918808 & 23 & $-1,56562$ & 22 & 0,131710 \\
\hline
\end{tabular}

In Tables 1 and 2 "Mean" - is an average figure, Std.Dv. - is an unbiased estimate of the standard deviation $s, t$ - is a sample value $t_{\text {observed }}, d f=n-1$ is the number of degrees of freedom of Student's distribution, $p$ ( $p$-level) - is the minimum value of the error probability of the first kind - the probability of mistakenly rejecting the main hypothesis in the observation sample $z_{1}, \ldots, z_{n}$. The threshold value of the probability of mistakenly rejecting the main hypothesis in Table 1 is equal to 0,319351 , therefore, at any smaller level of significance the hypothesis $H_{\mathrm{T} 0}: \theta_{\mathrm{T}}=0$ should be taken and it should be concluded that medium-chain fatty acids do not lead to variations in the number of triglycerides in an hour after testing. In Table 2 the threshold value of the probability of mistakenly rejecting the main hypothesis is equal to 0,13171 , which also allows to conclude that the number of triglycerides not changed and in two hours after testing in any smaller significance level.

In Tables 3 and 4 the threshold value of significance level (second and third rows) is given for comparison when checking two other main hypothesis $H_{\mathrm{T} 0}: \theta_{\mathrm{T}}=\theta_{m 0}$ where $\theta_{m 0}$ takes different nonzero values in the top rows of Tables 3 and 4 . If $\theta_{m 0}<0$ (Table 3 ) then we validate the hypothesis about the decrease in triglycerides after the test on the value $\theta_{m 0}$ if $\theta_{m 0}>0$ (Table 4 ) about increase.

Table 3. The threshold value of significance level when checking $\theta_{m 0}<0$.

\begin{tabular}{|l|l|l|l|l|}
\hline$\theta, \mathrm{mmol} / \mathrm{l}$ & $-0,5$ & $-0,2$ & $-0,1$ & $-0,05$ \\
\hline triglycerides after an hour & 0 & $9 \cdot 10^{-5}$ & 0,07 & $\mathbf{0 , 6 7}$ \\
\hline triglycerides after two hours & 0 & $10^{-4}$ & $5 \cdot 10^{-3}$ & 0,03 \\
\hline
\end{tabular}

Table 4. The threshold value of significance level when checking $\theta_{m 0}>0$.

\begin{tabular}{|l|l|l|l|l|}
\hline$\theta, \mathrm{mmol} / \mathrm{l}$ & 0,05 & 0,1 & 0,2 & 0,5 \\
\hline triglycerides after an hour & 0,02 & $7,5 \cdot 10^{-4}$ & $10^{-6}$ & 0 \\
\hline triglycerides after two hours & 0,43 & $\mathbf{0 , 9 7}$ & 0,15 & $10^{-6}$ \\
\hline
\end{tabular}

Table 3 shows that the maximum value of $p$-level, equal to 0,67 , obtained for $\theta_{m 0}=-0,05$ in assessing changes in the number of triglycerides in an hour after testing. In table 4 we can see that $p$-level takes the maximum value of 0,97 , when $\theta_{m 0}=0,1$ in that case, when the estimated the changing amount of triglycerides in two hours after testing. It should be noted that, according to the medical standard, the reduction of triglycerides by $0,05 \mathrm{mmol} / 1$ or increasing it by $0.1 \mathrm{mmol} / 1$ do not go beyond the norm, therefore, such changes can be considered insignificant. 
Next the main hypothesis $H_{l 0}: \theta_{l}=0$ meaning that the amount of lipase in the blood of patients on average has not changed under the influence of medium-chain fatty acids is being checked. The results of applying the student's t-test for samples of values of lipase in an hour and two hours after the test patients is shown in table 5 and table 6 , respectively. Since the $p$-levels are extremely high: $p=0,82$ an hour after the test and $p=0,93$, two hours after testing, it can be assumed that the using medium-chain fatty acids in clinical nutrition does not lead to an increase in the quantity of lipase in the blood of patients.

Table 5. The results of applying the student's t-test for samples of values of lipase in an hour after the test patients

\begin{tabular}{|l|l|l|l|l|l|}
\hline \multirow{2}{*}{ Zero hypothesis } & \multicolumn{5}{|l|}{ T-test for Dependent Samples } \\
\cline { 2 - 7 } & Mean & Std. Dv. & $\mathrm{t}$ & $\mathrm{df}$ & $\mathrm{p}$ \\
\hline $\begin{array}{l}\text { lipase on an empty } \\
\text { stomach }\end{array}$ & 63,03704 & 60,51286 & & & \\
\hline lipase after an hour & 60,33333 & 47,64371 & 0,232306 & 26 & 0,818119 \\
\hline
\end{tabular}

Table 6. The results of applying the student's t-test for samples of values of lipase in an two hours after the test patients

\begin{tabular}{|l|l|l|l|l|l|}
\hline \multirow{2}{*}{ Zero hypothesis } & \multicolumn{4}{l|}{ T-test for Dependent Samples } \\
\cline { 2 - 7 } & Mean & Std. Dv. & $\mathrm{t}$ & $\mathrm{df}$ & $\mathrm{p}$ \\
\hline $\begin{array}{l}\text { lipase on an empty } \\
\text { stomach }\end{array}$ & 63,03704 & 60,51286 & & & \\
\hline lipase after two hours & 61,77778 & 59,09792 & 0,084367 & 26 & 0,933411 \\
\hline
\end{tabular}

In Tables 7 and 8, the thresholds of significance (second and third rows) are shown when checking other main hypotheses: $H_{l 0}: \theta_{l}=\theta_{l 0}$ where $\theta_{l 0}$ takes different nonzero values in the top rows of Tables 7 and 8 . If $\theta_{l 0}<0$ (Table 7) then the hypothesis about the decrease in the level of lipase after testing on the value of $\theta_{l 0}$ is being tested, if $\theta_{l 0}>0$ then about the increasing it (Table 8). You can see that the $p$-levels when testing the null hypothesis (expressed in bold) exceeds all other values, therefore, the conclusion that the use medium-chain fatty acids in clinical nutrition does not lead to an increase in the quantity of lipase in the blood of patients is not doubted.

Table 7. The threshold value of significance level when checking $\theta_{l 0}<0$

\begin{tabular}{|l|l|l|l|l|}
\hline$\theta, \mathrm{u} / \mathrm{l}$ & -50 & -30 & -20 & -10 \\
\hline lipase after an hour & $4 \cdot 10^{-4}$ & 0,03 & 0,15 & 0,5 \\
\hline lipase after two hours & $3 \cdot 10^{-3}$ & 0,07 & 0,2 & 0,6 \\
\hline
\end{tabular}

Table 8. The threshold value of significance level when checking $\theta_{10}>0$

\begin{tabular}{|l|l|l|l|l|l|}
\hline$\theta, \mathrm{u} / \mathrm{l}$ & 0 & 10 & 20 & 30 & 50 \\
\hline lipase after an hour & $\mathbf{0 , 8}$ & 0,3 & 0,6 & $9 \cdot 10^{-3}$ & $10^{-4}$ \\
\hline lipase after two hours & $\mathbf{0 , 9}$ & 0,5 & 0,17 & 0,05 & $10^{-3}$ \\
\hline
\end{tabular}


Nonparametric methods of mathematical statistics were also used to test hypotheses $H_{\mathrm{T} 0}: \theta_{\mathrm{T}}=0, H_{l 0}: \theta_{l}=0$. The $p$-level of the Wilcoxon test for comparing the mathematical expectations of the samples $\omega_{1}, \omega_{2}, \ldots, \omega_{n}$ and $\chi_{1}, \chi_{2}, \ldots, \chi_{n}$ was 0,35 , and the $p$-level of the sign criterion was 0,83 . This confirms the conclusion that the use medium-chain fatty acids in clinical nutrition does not lead to an increase in triglycerides and lipase in the blood of patients.

\section{Conclusion}

Patients with a diagnosis of "chronic pancreatitis", often face abdominal pain, the symptom caused by consumption of products, which include both vegetable and animal fats. The reason of the syndrome is the disorder of exocrine pancreas and, as the food is digested much worse, it could be led to flatulence and diarrhea. In patients with chronic pancreatitis it is also frequently observed the protein-energy malnutrition and underweight.

The role of fats in the functioning of a human body cannot be underestimated. The reduction or absence of fat in the diet leads to disruption of energy and plastic of body functions. In violation of its intestinal absorption, for example, doctors implement some dairy products containing medium-chain fatty acids in everyday diet. There have been conducted some studies which have shown that the food with medium-chain fatty acids is well absorbed by patients with various disorders of the gastrointestinal tract and its application is recommended in cases of clinical nutrition.

The problem is the deficiency of knowledge about the influence of medium-chain fatty acids on functioning of the pancreas and its external non-secretory activity in chronic pancreatitis in the medical literature (Chekhonina, 2017).

The statistical analysis of changes in the number of lipase and triglycerides in the biochemical analysis of blood of patients with chronic pancreatitis after eating a meal containing medium-chain fatty acids has shown that dysfunction of the pancreas in these patients are generally not observed. Clinical practice has shown that more than $85 \%$ of patients are well responded to the testing of medium-chain fatty acids. Introduction in the diet the foods containing medium-chain fatty acids will help to solve the problem of energy insufficiency in chronic pancreatitis and increase the body mass index in these patients with no adverse impact on the pancreas.

\section{References:}

Chekhonina, Yu., Pilipenko, V., Shakhovskaya, A., Gapparov, K. 2017. Study of Actual Nutrition of Patients with Chronic Pancreatitis. Issues of Nutrition, 7(4), 22-25.

Gazaryan, V.A., Ivanitskaya N.V., Pyt'ev, Yu.P., Shakhovskaya, A.K. 2006. Vestnik of the Moscow University. Physics and Astronomy, 3, 12.

Gazaryan, V.A., Ilyushin, V.L., Pyt'ev, Yu. P., Shakhovskaya A.K. 2005. Vestnik of the Moscow University. Physics and Astronomy, 4, 3. 
Gazaryan, V.A., Pyt'ev, Y.P., Rosnitskiy, P.B. 2014. A Comparative Analysis of the Efficiency of Probabilistic and Possibilistic Algorithms for Medical Diagnostics. Moscow University Physics Bulletin, 69(3), 210-217.

Gazaryan, V.A., Matveeva, T.V., Chekhonina, Yu.G., Shakhovskaya A.K. 2014. On Theoretical-Possibilistic Methods of Analysis of the Effectiveness of Treatment. Intelligent systems. Theory and applications, 14(1-4), 107-122.

Gazaryan, V.A., Pyt'ev, Y.P., Rosnitskiy, P.B. 2015. Probabilistic and Possibilistic Methods of Making a Medical Diagnosis. Computer Implementation, Software System. Intelligent Systems. Theory and Applications, 18(4), 15-36.

Pyt'ev, Yu.P. 2018. The Likelihood or Possibility and Subjective Modeling in Scientific Research. Mathematical and Empirical Foundations and Applications. Moscow Fizmatlit. 